\title{
The Impact of Bisphenol A and Phthalates on Allergy, Asthma, and Immune Function: a Review of Latest Findings
}

\author{
Lacey Robinson $^{1} \cdot$ Rachel Miller $^{1,2,3,4}$
}

Published online: 4 September 2015

(C) Springer International Publishing AG 2015

\begin{abstract}
In recent years, the impact of environmental exposure to chemicals and their immunological effects, including the development of allergy, has been a topic of great interest. Epidemiologic studies indicate that exposure to endocrinedisrupting chemicals produced in high volumes, including bisphenol A (BPA) and phthalates, is ubiquitous. The links between their exposure and the development of allergy, asthma, and immune dysfunction have been studied in vitro, in vivo, and through human cohort studies. The purpose of this review is to examine the current body of research and to highlight deficits and strengths of current findings. Emerging science indicates that deleterious immunologic changes, including increased propensity to develop wheeze, allergy, and asthma after dietary and inhalation exposure to these chemicals, may be occurring.
\end{abstract}

This article is part of the Topical Collection on Synthetic Chemicals and Health

Rachel Miller

rlm14@cumc.columbia.edu

Lacey Robinson

1br2129@cumc.columbia.edu

1 Department of Medicine, Columbia University Medical Center, MH 6C-12, New York, NY 10032, USA

2 Department of Pediatrics, Columbia University Medical Center, New York, NY, USA

3 Department of Environmental Health Sciences, Columbia University Medical Center, New York, NY, USA

4 Division of Pulmonary, Allergy and Critical Care Medicine, Department of Medicine, Columbia University Medical Center, PH8E-101B, New York, NY 10032, USA
Keywords Bisphenol A $\cdot$ Phthalates $\cdot$ Allergy $\cdot$ Asthma . Immunotoxicity $\cdot$ Wheeze

$\begin{array}{ll}\text { Abbreviation } \\ \text { BBzP } & \text { Butyl benzyl phthalate } \\ \text { BPA } & \text { Bisphenol A } \\ \text { CCCEH } & \text { Columbia Center for Children's Environmental } \\ & \text { Health } \\ \text { DBP } & \text { Dibutyl phthalate } \\ \text { DEHP } & \text { Diethyl hexyl phthalate } \\ \text { DEP } & \text { Diethyl phthalate } \\ \text { DIDP } & \text { Diisodecyl phthalate } \\ \text { DINP } & \text { Diisononyl phthalate } \\ \text { DMP } & \text { Dimethyl phthalate } \\ \text { DnBP } & \text { Di- } n \text {-butyl phthalate } \\ \text { DNOP } & \text { Di- } n \text {-octyl phthalate } \\ \text { EDC } & \text { Endocrine-disrupting chemical } \\ \text { FeNO } & \text { Fractional exhaled nitric oxide } \\ \text { GALT } & \text { Gut-associated lymphoid tissue } \\ \text { iNOS } & \text { Inducible nitric oxide synthase } \\ \text { MBzP } & \text { Mono benzyl phthalate } \\ \text { NHANES } & \text { National Health and Nutrition Examination } \\ & \text { Survey } \\ \text { OVA } & \text { Ovalbumin } \\ \text { PVC } & \text { Polyvinyl chloride } \\ \text { ROS } & \text { Reactive oxygen species } \\ \text { Th } & \text { T helper } \\ \text { TSLP } & \text { Thymic stromal lymphopoietin } \\ \end{array}$

\section{Introduction}

According to the World Health Organization's report in 2007, at least $20 \%$ of the world's population was estimated to suffer 
from an allergic condition (http:/www.euro.who.int/ data/ assets/pdf_file/0012/96996/3.1.pdf?ua=1). Over recent years, the production and use of plastics and polycarbonates, which frequently contain bisphenol A (BPA) and phthalates, have risen dramatically. In light of these observations, the potential impact of exposure to these chemicals on the rising prevalence of atopy and allergy becomes an intriguing question. In this review, we will examine the recent data on BPA and phthalates as they pertain to the development of allergy and perturbed immunologic health, including the sources of exposure, proposed mechanisms of action, and compelling human cohort studies.

\section{Bisphenol A}

BPA is a 4,4-isopropylidenediphenol 2,2-bis(4-hydroxyphenyl)propane. It contains two functional phenol groups that allow the chemical to interact with estrogen and androgen receptors as both an agonist and antagonist [1]. This classifies BPA as a xenoestrogen (environmental estrogen). Its ability to stimulate the estrogen receptor further classifies it as an endocrinedisrupting chemical (EDC) [2, 3]. The estrogenic activity of BPA has been estimated to be $1 / 1000$ to $1 / 10,000$ of that of $17 \beta$-estradiol [4].

BPA is contained in polycarbonate plastics and epoxy resins and is used in the production of polyvinyl chloride [5]. Items containing BPA include children's toys, dental sealants, lining of water pipes, food and beverage containers such as plastic water bottles, food packaging, and the inner coating of cans and bottles $[2,6,7]$. It estimated that, each year, greater than 15 billions pounds of BPA are produced and over 100 tons are released into the atmosphere [3]. The primary route of exposure of BPA is oral, mainly from eating or drinking from products containing BPA. Other potential exposures include inhalation from dust particles and atmospheric exposure [1]. Once the compound is introduced via the oral route, it is absorbed in the gastrointestinal tract and undergoes hepatic metabolism including oxidation and hydrolysis, which leads to the production of several metabolites including BPA monosulfate, BPA glucuronide, and BPA disulfate [8]. These metabolites then undergo conjugation and can be excreted in the urine and feces [1]. The presence of BPA and its metabolites can be measured in bodily fluids, including urine and serum, by using the combined techniques of solid-phase extraction coupled with isotope dilution high-performance liquid chromatography and mass spectrometry [9]. Small studies have estimated the half-life of excretion of urinary BPA to approximate 4-5 h [10]. Subsequent pharmacokinetic analyses suggest that the rate of excretion of BPA is not greatly affected by fasting, implying that either BPA is excreted slowly from the body or there exists other significant areas of exposure beyond dietary routes [9, 10].

Epidemiologic studies performed in multiple countries found widespread exposure to BPA. For example, a study from Korea published in 2011 showed that $99.8 \%$ of the adult population has detectable urinary BPA. A higher concentration of urinary BPA was found in those who resided in rural areas than urban areas. They did not find evidence of significantly different levels of BPA in the urine based on sex, educational level, income, or body mass index [11]. A study of 1016 residents of Sweden, all 70 years or older, examined the serum for levels of BPA and several phthalate metabolites and again confirmed widespread exposure [12]. In the USA, the National Health and Nutrition Examination Survey (NHANES) study conducted from 2003 to 2004, levels tended to be higher among children and adolescents than adults [13]. A large cross-sectional study analyzed urine samples among 1890 Canadian women in the Maternal-Infant Research on Environmental Chemicals (MIREC) study cohort in their first trimester of pregnancy for the presence of BPA or its metabolite glucuronide of BPA. BPA or its metabolites were detected in up to $95 \%$ of urine samples. The highest concentrations of these compounds were measured in the urine of women who were less than 25 years of age, classified as low socioeconomic status or current smokers [14]. A review of greater than 80 exposure and toxicology studies again confirmed the ubiquitous exposure to BPA as measured by the presence of conjugated BPA in the vast majority of urine samples. In addition, levels of free unconjugated and, therefore, active form of BPA were often detected at levels of nanograms per millimeter in serum samples [9].

\section{Phthalates}

Phthalates are synthetic diesters of phthalic acid-dialkyl or alkyl/aryl esters of 1,2-benzenedicarboxylic acid [15]. The chemical structures of each individual phthalate vary, mostly according to their side chains and molecular weight. They can be grouped into two broad categories: low molecular weight and high molecular weight. Low-molecular-weight phthalates include dimethyl phthalate (DMP), diethyl phthalate (DEP), and dibutyl phthalate (DBP). High-molecular-weight phthalates include diethyl hexyl phthalate (DEHP), diisononyl phthalate (DINP), diisodecyl phthalate (DIDP), and benzyl butyl phthalate (BBzP) [15].

Phthalates are similarly produced in high volumes and are used as plasticizing agents. Low-molecular-weight phthalates are commonly found in cosmetics and personal care products, whereas high-molecular-weight phthalates are mostly associated with plastics, particularly polyvinyl chloride (PVC) containing building materials [16-18]. They serve to increase the stability and flexibility of compounds. Dietary exposures are likely the most common source of exposure, especially to the high-molecular-weight phthalates, and frequently occur due to exposure to food and beverage packaging containing PVC $[19,20 \bullet \bullet, 21]$. Inhalation exposure occurs through dust from vinyl flooring, carpet backing, and consumer products such as 
fragrances, dryer sheets, air fresheners, and sunscreens [22, 23]. The phthalate most often linked to indoor air and dust is DEHP, followed by BBzP [24]. Exposure through dermal contact also occurs with the use of phthalate-containing personal care products such as sunscreens, lotions, and cosmetics [25•]. Environmental exposures to phthalates are increased further due to the lack of covalent binding of phthalates in compounds; this property allows phthalates to leach out of the products and into the air, food, beverage, or personal care product [26]. Once phthalates enter the body, they are metabolized to their monoester forms through a variety of enzymatic conversions. These monoesters and other metabolites are then excreted in the urine [15]. Phthalates and their metabolites can be measured in the urine through a combination of solid-phase extraction coupled with high-performance liquid chromatography-isotope dilution and tandem spectrometry [20••].

As with BPA, the personal exposure to phthalates appears ubiquitous. According to the CDC's Fourth National Report on Human Exposure to Environmental Chemicals of urine samples from 2636 participants in the NHANES study, there was evidence of phthalate metabolites in the urine of almost all participants http://www.cdc.gov/exposurereport/pdf/ fourthreport.pdf). A study of 200 women and their newborn infants found evidence of phthalate exposure in cord blood and meconium [27]. A study of exposures in Europe found high levels of phthalate metabolites common to products such as makeup, shampoo, and lotion (including dimethyl phthalate (DMP), DEP, BBzP, DINP, and DIDP) in the urine of women [26]. Our research group at the Columbia Center for Children's Environmental Health (CCCEH) detected levels of phthalates among almost all children. Interestingly, $\mathrm{CCCEH}$ found higher levels of urinary phthalate metabolites of BBzP, di- $n$-butyl phthalate (DnBP), DEHP, and DEP among boys when compared to girls, but levels did not differ by age [20••].

\section{Links to Allergy and Asthma}

There has been growing interest in the effects of exposure to both BPA and phthalates on the alteration of immune function and the development of allergic diseases and asthma. To begin to clarify the link between the environmental exposure and alteration of immune function, we will first examine mechanistic studies followed by cross-sectional and prospective cohort studies.

\section{In Vitro and Ex Vivo Studies}

\section{Bisphenol A}

The mechanisms by which BPA predisposes to immune deregulation including allergy have not yet been elucidated; only scant direct mechanistic links have been detected. For example, two studies examined the ability of BPA to activate proallergic $\mathrm{T}$ helper (TH) cytokines. One in vitro study showed that BPA upregulated the $\mathrm{TH} 2$ pathway and production of interleukin (IL)-4 [28]. In another in vitro study examining the effect on dendritic cells after exposure to BPA versus $17-\beta$ estradiol, both with administration of tumor necrosis factor (TNF) alpha, BPA induced greater production of proallergic TH2 chemokines and cytokines, including $\mathrm{CC}$ chemokine ligand (CCL)-1, IL-10, IL-5, and IL-13. The expression of Th2 transcription factor GATA-binding protein 3 (GATA3) also was increased [4].

In vitro studies indicate that BPA acts on the estrogen and androgen receptors [29, 30]. Its activation of estrogen receptors has been well documented in studies that showed effects on gene expression, activation of the nuclear estrogen receptor-dependent signaling pathways, and selective estrogen receptor modulation $[31,32]$. As an environmental estrogen, BPA may enhance antigen presentation and induce the degranulation of mast cells [33] or promote dendritic cell maturation [34]. BPA also has aromatase-like activity with the ability to convert testosterone to estrogen and to activate the aryl hydrocarbon receptor involved in synthesis and degradation of steroid hormones [35]. These BPA-estrogen receptor interactions have been proposed as possible explanations underlying some of the epidemiological associations between estrogen activity and asthma. These include studies that have shown a rise in rates and severity in asthma as women enter puberty and into adulthood [33]. The severity of asthma has been linked to changes in estrogen levels during the menstrual cycle with one study indicating that up to $40 \%$ of asthmatic women are experiencing exacerbations during the perimenstrual period [36]. Furthermore, a Danish study showed that ever using exogenous estrogen in the form of hormone replacement therapy was associated with an increased risk of asthma-related hospitalizations (hazard ratio 1.46; $95 \%$ confidence interval (CI) 1.21-1.76) [37]. However, due to the paucity of research in this area, no clear mechanism of action has been defined for BPA that fully explains its ability to alter allergic or other immune functions.

\section{Phthalates}

While not fully characterized, there has been more investigation into the cellular mechanisms of phthalate effects on allergy. Diethyl phthalate (DEP) also can induce the production of pro-allergic TH-2 cytokines including IL-4 and TNF- $\alpha$ in human macrophages [38]. When human macrophages are exposed to the diethyl hexyl phthalate (DEHP), the production of non-allergic pro-inflammatory chemokines and cytokines in human macrophages was increased [39]. DEHP can cause mast cell to degranulate and release histamine and betahexoaminidase [40]. 


\section{Animal Models}

\section{Bisphenol A}

In murine studies of the effect of BPA on the development of allergic airway inflammation, prenatal exposure to BPA $(0$, $0.5,5,50$, or $500 \mu \mathrm{g} \mathrm{BPA} / \mathrm{kg} /$ day) induced variable effects on allergic lung inflammation for the offspring, depending on route of exposure and sex of the offspring. Evidence of increased allergic airway inflammation in those mice exposed prenatally and throughout life to BPA was not evident [41]. In mice exposed during weaning to BPA (50 ng, $50 \mu \mathrm{g}$, or $50 \mathrm{mg}$ of BPA $/ \mathrm{kg}$ ) through the dam's diet, there was evidence of increased systemic inflammatory cytokines in the spleen and serum IgE without increased airway inflammation on bronchoalveolar lavage or histologic sections of lung parenchyma [42].

Effects of BPA on ovalbumin-induced allergic airway inflammation reportedly varied based on timing and duration of exposure. Specifically, mice were exposed to either water containing $5 \mu \mathrm{g} / \mathrm{ml}$ of BPA or regular water during two distinct time periods - prenatally and perinatally through age 21 days or from birth through age 9 weeks. No significant difference in the development of allergic asthma phenotype was seen in mouse pups exposed to BPA via drinking water in the prenatal and perinatal period. However, mice exposed to BPA from birth through age 9 weeks developed higher levels of airway eosinophils, increased airway hyperreactivity, and increased levels of serum IgE [43]. Paradoxical effects were observed if BPA intake occurred only during the sensitization period [43]. Some of these results are corroborated in another experimental study that found that the mouse pups who displayed the asthma phenotype, defined as presence of airway eosinophilia and airway hyperreactivity, were those that were exposed both during the prenatal and postnatal periods to $10 \mu \mathrm{g} / \mathrm{ml}$ of BPA in the maternal drinking water. Those only exposed in the postnatal period did not exhibit the asthma phenotype [44•]. Hence, ultimately, environmental exposure to BPA may be linked to the development of airway inflammation and possibly even allergic sensitization; however, the effects of BPA exposure are likely modified by time period and by duration of exposure.

\section{Phthalates}

The majority of animal model studies on phthalates have focused on one major phthalate, DEHP, and its metabolites. Multiple studies have suggested DEHP's role as an adjuvant leading to an enhanced allergic responses in vivo. In one, DEHP's effect on follicular T cells and the allergic response in mice following exposure to DEHP gavage at doses of 0,30 , 300 , and $3000 \mu \mathrm{g} / \mathrm{kg}$ with ovalbumin (OVA) vs sham sensitization were studied. Those mice exposed to DEHP and OVA sensitized had increased levels of IgE, IgG1, IL-21, and IL-4, supporting the role of DEHP as an adjuvant [45]. In another, mice sensitized to OVA in the presence or absence of DEHP $(30,300$, and $3000 \mu \mathrm{g} / \mathrm{kg} /$ day $)$ via oral gavage were compared. Those exposed to DEHP + OVA exhibited higher levels of proallergic IgE, IL-4, interferon gamma (IFN- $\gamma$ ), and eosinophilia when compared to those mice exposed to OVA alone $(p<0.05)$ [46]. In experimental studies of the links between perinatal effects of exposure to DEHP and the development of airway inflammation, mice were exposed during gestation and for 21 days in the postnatal period to $2500 \mathrm{ppm}$ DEHP via the diet. Airway inflammation was assessed by measurements of mucus and inducible nitric oxide synthase (iNOS), a marker of airway inflammation. Paradoxically, exposure to DEHP was associated with protection from airway inflammation [47]. Dermal exposure to another phthalate, dibutyl phthalate (DBP), also upregulated TH-2 cytokines via increases in thymic stromal lymphopoietin (TSLP) in lymph node cells [48]. In a model of allergic rhinitis, mice sensitized to OVA with and without DEHP did not differ in levels of neutrophils or eosinophils in the nasal mucosa, but the additional exposure to DEHP did increase the production of IL-13 [49].

\section{Other Animal Models of Immunotoxicity}

BPA's adverse immunological effects do not seem limited to asthma or allergic airway inflammation in mice. For example, rats exposed to oral BPA at doses of $0.5,5$, or $50 \mu \mathrm{g} / \mathrm{kg} /$ day or vehicle control in the perinatal period from 15 th day of gravidity through weaning were assessed for perturbations in the development of oral tolerance. Higher titers of anti-OVA IgG, splenic IFN- $\gamma$, and activated CD $4{ }^{+} \mathrm{CD} 444^{\text {high }} \mathrm{CD} 62 \mathrm{~L}^{\text {low }} \mathrm{T}$ lymphocytes were found in OVA-tolerated and OVA-immunized animals, depending on dose. Following oral challenge with OVA among the tolerized animals, colonic inflammation developed with greater neutrophils and IFN- $\gamma$ and reduced levels of TGF- $\beta$, suggestive of abnormal maturation of gutassociated lymphoid tissue (GALT) [50•]. Similarly, OVAspecific $\mathrm{T}$ cell receptor transgenic mice exposed to BPA at doses of $0,0.1$, or $1 \mathrm{ppm}$ during pregnancy and weaning demonstrated altered $\mathrm{T}$ regulatory (i.e., forkhead box P3 (Foxp3) activity after OVA was administered to the pups on postnatal days 14,16 , and 18 . The pups exposed to BPA in the perinatal period had decreased levels of immunoregulatory $\mathrm{CD} 4{ }^{+} \mathrm{CD} 25^{+} \mathrm{Foxp}^{+} \mathrm{T}$ cells with higher titers of OVAspecific IgG1, IgG2a, IFN- $\gamma$, and IL-13 compared to nonexposed offspring after induction of oral tolerance $(p<0.05$ for all outcomes) [51].

DEHP also may exert pro-inflammatory effects through oxidative stress-mediated pathways. Mice were divided into six exposure groups: saline, DEHP at $30 \mathrm{mg} / \mathrm{kg}$ body weight/ day alone, OVA sensitization and challenge alone, DEHP + 
OVA, OVA + vitamin E $30 \mathrm{mg} / \mathrm{kg} /$ day, and DHEP + OVA + vitamin E. Those exposed to DEHP with OVA sensitization and challenge produced higher levels of reactive oxygen species (ROS) and malondialdehyde and lower levels of glutathione (all $p<0.05$ ), consistent with increased levels of oxidative stress. Those treated with vitamin $\mathrm{E}$ in addition to DEHP and OVA developed attenuated oxidative stress responses [52].

\section{Epidemiologic Studies}

Earlier clues to the contribution of BPA and phthalate exposure to the development of asthma and allergic disease were first derived from intriguing cross-sectional and case control studies. More recent prospective studies have informed us of many links and identified key deleterious time windows of exposure to chemicals (Table 1).

\section{Bisphenol A}

A cross-sectional study of the NHANES data examined the relationship between BPA and lung function among 661 children between the ages of 6-19 years of age. Higher levels of $\mathrm{BPA}$ in the urine were associated with a moderate decrease in small airway (average forced expiratory flow in percent during the mid $(25-75 \%)$ portion of the forced vital capacity (FVC) $(3.7 \%, 95 \%$ CI 1.0, 6.5) and lung function ( $\%$ forced expiratory volume at $1 \mathrm{~s}\left(\mathrm{FEV}_{1}\right) / \mathrm{FVC}(0.8 \%, 95 \% \mathrm{CI} 0.1$, 1.7) but not fractional exhaled nitric oxide (FeNO) [53]. A second NHANES study from 2003 to 2006 found no association between higher levels of BPA and allergy or allergic rhinitis [54].
Importantly, there are several prospective studies that further defined BPA's contribution to airway disease. A cohort study of 375 pregnant women during the third trimester and their children at ages 3, 5, and 7 examined effects of BPA measured in spot urine samples on the development of asthma. Paradoxically, a higher prenatal measure of BPA was related to a reduced report of wheeze at 5 years. As predicted, higher postnatal measures of BPA at age 3 were associated with more frequent wheeze at ages 5 and 6 (odds ratio (OR) $0.7 ; 95 \% \mathrm{CI}$ $0.5-0.9 ; p=0.02$ ). The detection of higher levels of urinary BPA at the age of 7 was associated with increased risk of wheeze at the age of 7 (OR 1.4; $95 \%$ CI 1.0-1.9; $p=0.04$ ). These results support that continued or postnatal exposure to BPA increased the likelihood of developing wheeze [55••].

Similarly, among a second cohort study of 657 pregnant women, prenatal BPA exposure was determined by spot urine BPA concentrations during the first and third trimesters and the development of eczema, allergy, asthma, wheeze, and chest infections including bronchiolitis in the children assessed. Relative risk (RR) of wheeze (RR 1.20; $95 \%$ CI, $1.03-1.40 ; p=0.02$ ), chest infection (RR 1.15 ; $95 \%$ CI 1.00 $1.32 ; p=0.05$ ), and development of bronchitis (RR 1.18; $95 \%$ CI 1.01-1.37; $p=0.04$ ) increased with every doubling in the concentration of urinary BPA for all age groups. The risk of asthma at age 7 was increased with increased levels or prenatal BPA [56]. A cohort of 398 mothers and children compared urinary BPA levels collected serially during the first and third trimesters, and children were assessed for the presence of wheeze via questionnaire every 6 months for 3 years. As found in previous studies, BPA was present in $>99 \%$ of maternal urine samples. Mothers with urine BPA concentrations greater than the median had children who were more likely to report wheeze at 6 months of age (adjusted OR 2.3; $95 \% \mathrm{CI}$

Table 1 BPA and phthalates on asthma-related outcomes: human cohort studies

\begin{tabular}{|c|c|c|c|c|c|}
\hline $\begin{array}{l}\text { Number of } \\
\text { participants }\end{array}$ & $\begin{array}{l}\text { Study } \\
\text { design }\end{array}$ & Measure(s) & Timing & Outcome(s) & Citation \\
\hline 1890 & Cross-sectional & Urinary BPA & First trimester of pregnancy & $\begin{array}{l}\text { Differences by age, socioeconomic } \\
\text { status, and smoking }\end{array}$ & Arbuckle [14] \\
\hline 661 & Cross-sectional & Urinary BPA & Children $16-19$ years & $\begin{array}{l}\text { Decreased lung function, no } \\
\text { difference in FeNO }\end{array}$ & Spanier [53] \\
\hline 375 & Prospective & Urinary BPA & $\begin{array}{l}\text { Third trimester of pregnancy; } \\
\text { children } 3,5,7 \text { years }\end{array}$ & $\begin{array}{l}\text { Prenatal BPA inversely associated } \\
\text { with wheeze at age } 3 \text {, childhood } \\
\text { BPA positively with wheeze }\end{array}$ & Donohue [54] \\
\hline 657 & Prospective & Urinary BPA & First and third trimesters of pregnancy & Wheeze, asthma, respiratory infections & Gascon [56] \\
\hline 398 & Prospective & Urinary BPA & $\begin{array}{l}\text { Second and third trimesters of } \\
\text { pregnancy }\end{array}$ & Wheeze at 6 months & Spanier [57] \\
\hline 405 & Cross-sectional & $\begin{array}{l}\text { BBzP and DEHP in } \\
\text { house dust }\end{array}$ & Children $3-8$ years & $\begin{array}{l}\text { BBzP, PVC flooring associated } \\
\text { with allergy. DEHP with asthma }\end{array}$ & Bornehag [58] \\
\hline 184 & Case control & DEHP in house dust & Children $2,3,5$, and 7 years & Allergy & Kolarik [59] \\
\hline 2325 & Cross-sectional & Urinary phthalates & Children over 6 years, adults & $\begin{array}{l}\text { MBzP, DEHP associated with } \\
\text { allergy in adults, not children. }\end{array}$ & Hoppin [60] \\
\hline 244 & Prospective & $\begin{array}{l}\text { Prenatal urinary } \\
\text { phthalates }\end{array}$ & $\begin{array}{l}\text { Third trimester of pregnancy, } \\
\text { children } 3,5,7 \text { years }\end{array}$ & Asthma & Whyatt $[20 \bullet \bullet]$ \\
\hline
\end{tabular}


$1.3,4.1)$, but not at age 3 years. A closer analysis of the data found that the higher urine concentrations of BPA at 16-week gestation, but not 26 weeks, were associated with development of wheeze (adjusted OR 1.2; $95 \%$ CI 1.0, 1.5) [57].

\section{Phthalates}

A cohort of children in Sweden (198 children with persistent allergic symptoms and 202 controls) was studied for links between exposure to phthalates, including BBzP and DEHP as measured in house dust and the development of allergy and asthma. Data were collected regarding the type of flooring material present in the children's homes, specifically PVC containing versus wood flooring. Higher levels of BBzP were measured in the house dust of allergic cases and among those with the diagnosis of rhinitis $(p=0.001)$ and eczema $(p=0.001)$. A higher concentration of DEHP in the dust was associated with diagnosis of asthma $(p=0.022)$. Not surprisingly, the presence of PVC flooring in the home also was associated with the diagnosis of eczema $(p=0001)$, rhinitis $(p=0.001)$, and asthma $(p=0.022)$ [58]. A similar case-control study in Bulgaria studied 102 children with wheeze, eczema, or rhinitis in the last year and 82 healthy controls all aged 2-7 years. Dust was collected from their homes and assessed for levels of phthalates including DMP, DEP, di- $n$ butyl phthalate (DnBP), BBzP, DEHP, and di- $n$-octyl phthalate (DnOP). Higher concentrations of DEHP were found in the house dust of allergic cases than controls (1.24 vs. $0.86 \mathrm{mg} / \mathrm{g}$ dust). The level of DEHP was related to episodes of wheeze within the last 12 months, and the analysis suggested that there is a dose-response relationship with DEHP exposure [59].

The data from the NHANES study from 2005 to 2006 were examined for the association between phthalates and allergic symptoms including asthma, wheeze, allergy, rhinitis, and hay fever among both adult and pediatric ( $>6$ years) populations. In the adult population, mono benzyl phthalate (MBzP) levels were associated with current asthma, wheeze, hay fever, and allergic rhinitis (OR 1.01-1.78). Inverse or insignificant associations were detected among the children [60] Within the Columbia Center for Children's Environmental Health (CCCEH) urban birth cohort, the relationship between levels of phthalate metabolites in the urine of 244 children aged 4 9 years and the association with airway inflammation as measured by the fractional excretion of nitric oxide (FeNO) were assessed. Metabolites of DEP and BBzP were associated with an increase in airway inflammation as measured by levels of FeNO. DEP increased FeNO by $6.6 \%$ (95 \% CI $0.5-13.1 \%)$, and BBzP increased FeNO by $8.7 \%$ (95 \% CI 1.9-16.0\%). Among the children who had reported wheeze during the study period, the association between $\mathrm{BBzP}$ and $\mathrm{FeNO}$ was stronger $(p=0.016)$. Levels of urinary phthalate metabolites were not associated with levels of IgE, suggestive of a nonatopic mechanism [61].

In an attempt to examine some of the other mechanisms by which phthalates may induce immunotoxicity, the association between phthalates and the levels of oxidative stress in pregnant women was examined in a nested case control study of 130 preterm births with 352 controls delivering in Boston, MA. Urine samples were collected at least four times during pregnancy, and levels of phthalate metabolites and markers of oxidative stress (8-hydroxydeoxyguanosine $(\mathrm{OHdG})$ and 8isoprostane) were measured. Those with increased levels of phthalates also had higher urinary makers of oxidative stress ( $p=0.001)$ [62]. Similarly, a prospective cohort study by some of the same investigators assessed 139 pregnant women in Puerto Rico with repeat measures during pregnancy of phthalate urinary metabolites, plasma biomarkers of inflammation, and urinary biomarkers of oxidative stress $(\mathrm{OHdG}$ and 8isoprostane). All of the phthalate metabolites were associated with the biomarkers of oxidative stress, but the larger percent increases were for associations of isoprostane and interquartile range increase in urinary MBP $(\% \Delta=49.7,95 \% \mathrm{CI}=32.0$ $69.8)$ and monoisobutyl phthalate (MiBP) $(\% \Delta=48.8,95 \%$ CI 30.4-69.8) concentrations. Few significant findings with high-molecular-weight, but not low-molecular-weight, metabolites and TNF- $\alpha$, C-reactive protein, IL- $1 \beta$, IL-6, and IL-10 were apparent [63].

There are several prospective studies that furthered our knowledge on phthalates and allergy and airway disease. In one, the risk of prenatal exposure to phthalates was examined for the development of respiratory tract infections, allergy, or asthma by the age of 7. Higher levels of DEHP metabolites were associated with the development of chest infections (RR $1.15,95 \%$ CI, $0.97-1.35 ; p=0.11$ ), bronchitis (RR 1.20; $95 \%$ CI 1.01-143; $p=0.04$ ), and wheeze (RR 1.25; $95 \%$ CI, 1.04 $1.50, p=0.02$ ). The risk of developing asthma by age 7 was associated with increased levels of MBzP and DEHP metabolites. MBzP was associated with an increase risk of wheeze ((RR, 1.15; $95 \%$ CI, 1.00-1.33; $p=0.05$ ) [56]. The Dampness in Buildings and Health study conducted in Sweden sought to determine if children exposed to phthalates through PVC flooring were more likely to develop asthma, rhinitis, or allergies. At the start of the study, they queried all children aged 16 years in the study area regarding baseline characteristics and qualities of the homes, including the presence of PVC flooring. Over the next 5 years, children were assessed for the development of asthma, rhinitis, or eczema via questionnaire. An association was found between the diagnosis of asthma and the presence of PVC flooring in both the child's bedroom (adjusted OR 1.52; $95 \%$ CI 0.99-2.35) and the parents' bedroom (adjusted OR 1.46; $95 \%$ CI 0.96-2.23). There was also a positive correlation with the number of rooms in the home containing PVC flooring and the incidence of asthma [64]. A second study continued to follow the children for an additional 
5 years resulting in 10 years of prospective data. The presence of PVC flooring in the child's bedroom again was associated with the development of asthma in the following 5-10 years (OR 1.49; $95 \%$ CI 0.92-2.42). Interestingly, the strongest association with the development of childhood asthma was the presence of the PVC flooring in the parents' bedroom (OR 1.75; $95 \%$ CI 1.08-2.83), implying that prenatal exposure to phthalates may be associated with delayed detrimental consequences [65].

Our group at CCCEH looked for a link between prenatal exposure to BBzP and the development of eczema. Levels of the main $\mathrm{BBzP}$ metabolite, $\mathrm{MBzP}$, were measured in spot urine samples from women in their third trimester of pregnancy. Ubiquitous exposure was again confirmed in this population as $\mathrm{MBzP}$ was found in $>99 \%$ of urine samples. Increased levels of maternal urinary MBzP were associated with the early onset of eczema $(\mathrm{RR}=1.52 ; 95 \%$ CI 1.21-1.91, $p=0.0003$ ), but not levels of immunoglobulin E [66]. Furthermore, CCCEH examined associations between asthma and phthalates BBzP, DnBP, DEHP, and DEP at age 5-11 years. An association was found between prenatal urinary levels of $\mathrm{BBzP}$ and DEP metabolites with the development of asthmalike symptoms and with the diagnosis of asthma during childhood. Notably, the risk of asthma was greater than $70 \%$ higher for the children of mothers who were found to have urinary concentrations of $\mathrm{BBzP}$ and $\mathrm{DnBP}$ in the third vs. first tertiles $[20 \bullet \cdot$.

\section{Conclusions}

In the past decade, researchers begun to examine the relationships between common chemical exposures, including BPA and phthalates, and their links to the development of allergy, asthma, and immune dysfunction. It is clear from multiple epidemiologic studies that human exposure to these chemicals is ubiquitous due to their use in a wide range of consumer products, food production, and building materials. The attempts at elucidating the mechanisms by which these chemicals are potentially immune modifying have been sparse, and their results are fairly inconclusive. While the biochemical effects of phthalates have been studied more extensively when compared to BPA, no clear consensus has been made about the underlying mechanisms of allergy and asthma, although DEHP has been studied more extensively in mouse models and most studies do seem to support some role as an adjuvant.

The studies performed in human subjects draw no clear conclusion about the roles that BPA and phthalates play in the rising incidence of allergy and asthma, but they appear to substantiate a link between these chemical exposures and asthma outcomes. Several studies found that increased exposure to BPA was associated with the development of wheeze or atopy. They also suggest that the timing and duration of exposure to BPA alter the clinical phenotype. Many of the studies are limited by the analysis of spot urine samples and use of self-reported questionnaires to determine the development of allergy, asthma, wheeze, or eczema. Many of the population-based human studies confirm the relationship with phthalates, specifically DEHP, with the increased risk of developing wheeze, asthma, and allergy. The associations between other phthalates and allergy are less clear. The most vulnerable time for exposure to these chemicals also has not yet been determined, although the prenatal period seems to be especially important.

Future research should focus on defining the mechanism of action of these chemicals and the dose at which these effects occur. It will be imperative to discern the key time period for exposure to these chemicals, as well as characterize the most susceptible populations in which immune changes take place. Ultimately, if the research continues to support the existence of a relationship between these chemicals and immune deregulation, it will be necessary to determine methods to limit further exposure to these chemicals.

Funding R01ES014393, RC2 ES018784.

\section{Compliance with Ethics Guidelines}

Conflict of Interest Lacey Robinson and Rachel Miller declare that they have no conflict of interest.

Human and Animal Rights and Informed Consent This article does not contain any new study data with human or animal subjects performed by any of the authors for purposes of this article.

\section{References}

Papers of particular interest, published recently, have been highlighted as:

- Of importance

•. Of major importance

1. Michalowicz J. Bisphenol A - sources, toxicity and biotransformation. Environ Toxicol Pharmacol. 2014;37(2):738-58.

2. Vandenberg LN et al. Human exposure to bisphenol A (BPA). Reprod Toxicol. 2007;24(2):139-77.

3. Vandenberg LN et al. Bisphenol-A and the great divide: a review of controversies in the field of endocrine disruption. Endocr Rev. 2009;30(1):75-95.

4. Guo $\mathrm{H}$ et al. Bisphenol A in combination with TNF-alpha selectively induces Th2 cell-promoting dendritic cells in vitro with an estrogen-like activity. Cell Mol Immunol. 2010;7(3):227-34.

5. Rosenfeld CS. Bisphenol A and phthalate endocrine disruption of parental and social behaviors. Front Neurosci. 2015;9:57.

6. Konieczna A, Rutkowska A, Rachon D. Health risk of exposure to bisphenol A (BPA). Rocz Panstw Zakl Hig. 2015;66(1):5-11. 
7. Vandenberg L et al. Human exposure to bisphenol A (BPA). Reprod Toxicol. 2007;24(2):139-77.

8. Matthews JB, Twomey K, Zacharewski TR. In vitro and in vivo interactions of bisphenol A and its metabolite, bisphenol A glucuronide, with estrogen receptors alpha and beta. Chem Res Toxicol. 2001;14(2):149-57.

9. Vandenberg LN et al. Urinary, circulating, and tissue biomonitoring studies indicate widespread exposure to bisphenol A. Environ Health Perspect. 2010;118(8):1055-70.

10. Stahlhut RW, Welshons WV, Swan SH. Bisphenol A data in NHANES suggest longer than expected half life, substantial nonfood exposure, or both. Environ Health Perspect. 2009;117(5):784-9.

11. Kim $\mathrm{K}$ et al. Urinary concentrations of bisphenol A and triclosan and associations with demographic factors in the Korean population. Environ Res. 2011;111(8):1280-5.

12. Olsén L et al. Circulating levels of bisphenol A (BPA) and phthalates in an elderly population in Sweden, based on the Prospective Investigation of the Vasculature in Uppsala Seniors (PIVUS). Ecotoxicol Environ Saf. 2012;75:242-8.

13. LaKind JS, Naiman DQ. Daily intake of bisphenol A and potential sources of exposure: 2005-2006 National Health and Nutrition Examination Survey. J Expos Sci Environ Epidemiol. 2011;21(3): 272-9.

14. Arbuckle TE et al. Exposure to free and conjugated forms of bisphenol $\mathrm{A}$ and triclosan among pregnant women in the MIREC cohort. Environ Health Perspect. 2015;123(4):277-84.

15. North ML et al. Effects of phthalates on the development and expression of allergic disease and asthma. Ann Allergy Asthma Immunol. 2014;112(6):496-502.

16. Buckley JP et al. Consumer product exposures associated with urinary phthalate levels in pregnant women. J Expo Sci Environ Epidemiol. 2012;22(5):468-75.

17. Carlstedt F, Jönsson B, Bornehag C. PVC flooring is related to human uptake of phthalates in infants. Indoor Air. 2013;23(1):32-9.

18. Duty SM et al. Personal care product use predicts urinary concentrations of some phthalate monoesters. Environ Health Perspect. 2005;113(11):1530-5.

19. Koch HM et al. Identifying sources of phthalate exposure with human biomonitoring: results of a $48 \mathrm{~h}$ fasting study with urine collection and personal activity patterns. Int J Hyg Environ Health. 2013;216(6):672-81.

$20 . \bullet$ Whyatt RM et al. Asthma in inner-city children at 5-11 years of age and prenatal exposure to phthalates: the Columbia Center for Children's Environmental Health Cohort. Environ Health Perspect. 2014;122(10):1141-6. Important study demonstrating the relationship between phthalate exposure and wheeze.

21. Rudel RA et al. Food packaging and bisphenol A and bis(2ethyhexyl) phthalate exposure: findings from a dietary intervention. Environ Health Perspect. 2011;119(7):914-20.

22. Just, A.C., et al., Vinyl flooring in the home is associated with children's airborne butylbenzyl phthalate and urinary metabolite concentrations. J Expo Sci Environ Epidemiol, 2015.

23. Dodson RE et al. Endocrine disruptors and asthma-associated chemicals in consumer products. Environ Health Perspect. 2012;120(7):935-43.

24. Kolarik B et al. The association between phthalates in dust and allergic diseases among Bulgarian children. Environ Health Perspect. 2008;116(1):98-103.

25. Li J et al. T-helper type-2 contact hypersensitivity of Balb/c mice aggravated by dibutyl phthalate via long-term dermal exposure. PLoS One. 2014;9(2):e87887. Mouse model of phthalates leading to immunotoxicity which highlights alternate exposure to phthalates including the dermal route.

26. Wormuth $\mathrm{M}$ et al. What are the sources of exposure to eight frequently used phthalic acid esters in Europeans. Risk Anal. 2006;26: 803-24.
27. Li L-X et al. Exposure levels of environmental endocrine disruptors in mother-newborn pairs in China and their placental transfer characteristics. PLoS One. 2013;8(5):e62526.

28. Tian X, Takamoto M, Sugane K. Bisphenol A promotes IL-4 production by Th2 cells. Int Arch Allergy Immunol. 2003;132(3):240-7.

29. Kuiper GG et al. Interaction of estrogenic chemicals and phytoestrogens with estrogen receptor beta. Endocrinology. 1998;139(10):4252-63.

30. Hiroi $\mathrm{H}$ et al. Differential interactions of bisphenol A and 17 betaestradiol with estrogen receptor alpha (ERalpha) and ERbeta. Endocr J. 1999;46(6):773-8.

31. Richter CA et al. Estradiol and bisphenol A stimulate androgen receptor and estrogen receptor gene expression in fetal mouse prostate mesenchyme cells. Environ Health Perspect. 2007;115(6): 902-8.

32. Welshons WV, Nagel SC, Saal FS. Large effects from small exposures. III. Endocrine mechanisms mediating effects of bisphenol A at levels of human exposure. Endocrinology. 2006;147(6):s56-69.

33. Bonds RS, Midoro-Horiuti T. Estrogen effects in allergy and asthma. Curr Opin Allergy Clin Immunol. 2013;13(1):92-9.

34. Uemura $\mathrm{Y}$ et al. 17 Beta-estradiol (E2) plus tumor necrosis factoralpha induces a distorted maturation of human monocyte-derived dendritic cells and promotes their capacity to initiate T-helper 2 responses. Hum Immunol. 2008;69(3):149-57.

35. Bonefeld-Jorgensen EC et al. Endocrine-disrupting potential of bisphenol A, bisphenol A dimethacrylate, 4-n-nonylphenol, and 4-n-octylphenol in vitro: new data and a brief review. Environ Health Perspect. 2007;115 Suppl 1:69-76.

36. Thornton $\mathrm{J}$ et al. Clinical characteristics of women with menstruallinked asthma. Respir Med. 2012;106(9):1236-43.

37. Bønnelykke $\mathrm{K}$ et al. Postmenopausal hormone therapy and asthmarelated hospital admission. J Allergy Clin Immunol. 2015;135(3): 813-816.e5.

38. Ochiai $\mathrm{S}$ et al. CD326(lo)CD103(lo)CD11b(lo) dermal dendritic cells are activated by thymic stromal lymphopoietin during contact sensitization in mice. J Immunol. 2014;193(5):2504-11.

39. Nishioka J et al. Di-(2-ethylhexyl) phthalate induces production of inflammatory molecules in human macrophages. Inflamm Res. 2012;61(1):69-78.

40. Kuo $\mathrm{CH}$ et al. Phthalates suppress type I interferon in human plasmacytoid dendritic cells via epigenetic regulation. Allergy. 2013;68(7):870-9.

41. Bauer SM et al. The effects of maternal exposure to bisphenol A on allergic lung inflammation into adulthood. Toxicol Sci. 2012;130(1):82-93.

42. O'Brien E et al. Perinatal bisphenol A exposure beginning before gestation enhances allergen sensitization, but not pulmonary inflammation, in adult mice. J Dev Orig Health Dis. 2014;5(2): 121-31.

43. Petzold S et al. Lifetime-dependent effects of bisphenol A on asthma development in an experimental mouse model. PLoS One. 2014;9(6):e100468.

44. Nakajima Y, Goldblum RM, Midoro-Horiuti T. Fetal exposure to bisphenol $\mathrm{A}$ as a risk factor for the development of childhood asthma: an animal model study. Environ Health. 2012;11:8. Excellent animal model of BPA exposure.

45. Han Y et al. Di-(2-ethylhexyl) phthalate adjuvantly induces imbalanced humoral immunity in ovalbumin-sensitized BALB/c mice ascribing to $\mathrm{T}$ follicular helper cells hyperfunction. Toxicology. 2014;324:88-97.

46. Guo $\mathrm{J}$ et al. Pulmonary toxicity and adjuvant effect of di-(2exylhexyl) phthalate in ovalbumin-immunized BALB/c mice. PLoS One. 2012;7(6):e39008.

47. Shin IS et al. Effects of maternal exposure to di(2ethylhexyl)phthalate (DEHP) during pregnancy on susceptibility to neonatal asthma. Toxicol Appl Pharmacol. 2014;274(3):402-7. 
48. Shigeno T et al. Phthalate ester-induced thymic stromal lymphopoietin mediates allergic dermatitis in mice. Immunology. 2009;128(1 Suppl):e849-57.

49. He M et al. Effects of airway exposure to di-(2-ethylhexyl) phthalate on allergic rhinitis. Immunopharmacol Immunotoxicol. 2013;35(3):390-5.

50. Menard $\mathrm{S}$ et al. Food intolerance at adulthood after perinatal exposure to the endocrine disruptor bisphenol A. Faseb J. 2014;28(11): 4893-900. Explores other mechanisms and BPA exposure leading to immunotoxicity and highlights the disruption of possible immune regulation.

51. Ohshima $\mathrm{Y}$ et al. Transmaternal exposure to bisphenol a modulates the development of oral tolerance. Pediatr Res. 2007;62(1):60-4.

52. You $\mathrm{H}$ et al. The adjuvant effect induced by di-(2-ethylhexyl) phthalate (DEHP) is mediated through oxidative stress in a mouse model of asthma. Food Chem Toxicol. 2014;71:272-81.

53. Spanier AJ, Fiorino EK, Trasande L. Bisphenol A exposure is associated with decreased lung function. J Pediatr. 2014;164(6): 1403-8.e1.

54. Clayton EM et al. The impact of bisphenol A and triclosan on immune parameters in the U.S. population, NHANES 20032006. Environ Health Perspect. 2011;119(3):390-6.

55.• Donohue KM et al. Prenatal and postnatal bisphenol A exposure and asthma development among inner-city children. J Allergy Clin Immunol. 2013;131(3):736-42. Important prospective human cohort showing the relationship between BPA exposure and the development of asthma.

56. Gascon M et al. Prenatal exposure to bisphenol A and phthalates and childhood respiratory tract infections and allergy. J Allergy Clin Immunol. 2015;135(2):370-8.
57. Spanier A et al. Prenatal exposure to bisphenol A and child wheeze from birth to three years. Environ Health Perspect. 2012;120(6): 916-20.

58. Bornehag CG et al. The association between asthma and allergic symptoms in children and phthalates in house dust: a nested casecontrol study. Environ Health Perspect. 2004;112:1393-97.

59. Kolarik B et al. The concentrations of phthalates in settled dust in Bulgarian homes in relation to building characteristic and cleaning habits in the family. Atmos Environ. 2008;42(37):8553-59.

60. Hoppin JA et al. Phthalate exposure and allergy in the U.S. population: results from NHANES 2005-2006. Environ Health Perspect. 2013;121(10):1129-34.

61. Just AC et al. Children's urinary phthalate metabolites and fractional exhaled nitric oxide in an urban cohort. Am J Respir Crit Care Med. 2012;186(9):830-37.

62. Ferguson KK et al. Urinary phthalate metabolites and biomarkers of oxidative stress in pregnant women: a repeated measures analysis. Environ Health Perspect. 2015;123(3):210-6.

63. Ferguson KK et al. Urinary phthalate metabolite associations with biomarkers of inflammation and oxidative stress across pregnancy in Puerto Rico. Environ Sci Technol. 2014;48(12):7018-25.

64. Larsson $\mathrm{M}$ et al. PVC — as flooring material — and its association with incident asthma in a Swedish child cohort study. Indoor Air. 2010;20(6):494-501.

65. Shu $\mathrm{H}$ et al. PVC flooring at home and development of asthma among young children in Sweden, a 10-year follow-up. Indoor Air. 2014;24(3):227-35.

66. Just AC et al. Prenatal exposure to butylbenzyl phthalate and early eczema in an urban cohort. Environ Health Perspect. 2012;120(10): 1475-80. 